\title{
Rapid Differentiation and Identification of Potential Severe Strains of Citrus tristeza virus by Real-Time Reverse Transcription- Polymerase Chain Reaction Assays
}

\author{
R. K. Yokomi, M. Saponari, and P. J. Sieburth
}

First author: United States Department of Agriculture-Agricultural Research Service (USDA-ARS), Parlier, CA 93648; second author: Istituto di Virologia Vegetale del CNR, Sezione di Bari e Dipartimento di Protezione delle Piante e Microbiologia Applicata dell'Universita di Bari, 70126, Italy; and third author: Bureau of Citrus Budwood Registration, Florida Department of Agriculture and Consumer Services, Division of Plant Industry, Winter Haven, FL 33881.

Accepted for publication 13 November 2009.

\begin{abstract}
Yokomi, R. K., Saponari, M., and Sieburth, P. J. 2010. Rapid differentiation and identification of potential severe strains of Citrus tristeza virus by real-time reverse transcription-polymerase chain reaction assays. Phytopathology 100:319-327.

A multiplex Taqman-based real-time reverse transcription (RT) polymerase chain reaction (PCR) assay was developed to identify potential severe strains of Citrus tristeza virus (CTV) and separate genotypes that react with the monoclonal antibody MCA13. Three strain-

genotypes; and probe CPi-T36-NS to identify isolates in an outgroup clade of T36-like genotypes mild in California. Total nucleic acids extracted by chromatography on silica particles, sodium dodecyl sulfatepotassium acetate, and CTV virion immunocapture all yielded high quality templates for real-time PCR detection of CTV. These assays successfully differentiated CTV isolates from California, Florida, and a large panel of CTV isolates from an international collection maintained in Beltsville, MD. The utility of the assay was validated using field isolates collected in California and Florida.
\end{abstract} specific probes were developed using intergene sequences between the major and minor coat protein genes $(\mathrm{CPi})$ in a multiplex reaction. Probe CPi-VT3 was designed for VT and T3 genotypes; probe CPi-T36 for T36
Additional keywords: decline, seedling yellows, stem pitting, strain differentiation.
Citrus tristeza virus (CTV) (Closteroviridae) is a phloemlimited, semipersistent aphid-transmitted virus. The virus exists in infected trees as heterogeneous populations, often with more than one genotype along with variants and defective RNAs $(1,8,15$, $17,24,32)$. This genetic complexity results in different symptoms among citrus cultivars. Strains characterized in a citrus host range index test are generally categorized as: mild, decline inducing death of scions grafted onto sour orange (SO) rootstock; and sweet orange stem pitting (OSP) and grapefruit stem pitting (GSP). OSP and GSP are damaging CTV isolates that cause defective phloem development resulting in greatly reduced growth and production. Isolates that cause stunting and chlorosis of grapefruit, sour orange, or lemon seedlings in the greenhouse are referred to as seedling yellows (SY) $(5,18)$. Thus, the ability to differentiate CTV strains is practical and important for the management of diseases caused by CTV.

Spread of CTV in Central California has increased significantly over the past few years $(33,34)$ and local efforts to suppress the virus by survey and eradication have become too costly and extremely difficult. Routine surveys and monitoring of CTV in California have been based on enzyme-linked immunosorbent assay (ELISA) using universal CTV antiserum, whereas Florida used MCA13 antibody which generally detects all CTV strains except T30 (19,20). However, MCA13 does not distinguish isolates that cause only decline from isolates that also may cause stem pitting or mild symptoms, creating a need for more selective

Corresponding author: R. K. Yokomi; E-mail address: ray.yokomi@ars.usda.gov

doi:10.1094/PHYTO-100-4-0319

This article is in the public domain and not copyrightable. It may be freely reprinted with customary crediting of the source. The American Phytopathological Society, 2010. probes to rapidly discriminate CTV strains among MCA13 reactive isolates.

Other detection procedures have been developed to differentiate strains which include, but are not limited to, single-strand conformation polymorphism $(23,25)$; restriction fragment polymorphism length analysis (7); reverse transcription (RT)-polymerase chain reaction (PCR) assay with selective primers $(4,11,13)$; and hybridization with specific probes $(6,9)$. Saponari and Yokomi (29) developed a quantitative real-time RT-PCR (qRT-PCR) assay to identify some CTV isolates based on $3^{\prime}$ end sequences. RuizRuiz et al. (27) described a qPCR assay to differentiate CTV strains using TaqMan LNA probes designed from sequences in the $5^{\prime}$ portion of the CTV genome.

Rapid identification of genetic diversity of CTV by qPCR can aid efforts to manage CTV by identifying potentially virulent isolates that growers and regulatory agencies can target for CTV disease management decisions. We developed a qPCR assay using specific TaqMan probes for simultaneous detection and differentiation of CTV strains within $2 \mathrm{~h}$ which allows rapid assessment of potential virulent CTV strains. This method is under consideration for use by the Central California Tristeza Eradication Agency (CCTEA), Tulare, CA, to select CTV-infected trees that should be removed to eliminate potential severe strain inoculum sources. The Bureau of Citrus Budwood Registration (BCBR), Florida Department of Agriculture and Consumer Services, Winter Haven, FL, is also considering using this qPCR method to identify severe CTV strains found contaminating registered budwood source trees. This is particularly important in Florida because the efficient CTV vector, Toxoptera citricda, is well established and accelerates spread of virulent CTV strains (9). This work was designed to validate this procedure. 


\section{MATERIALS AND METHODS}

CTV isolates. The assays were developed and assessed with 56 California CTV isolates maintained by the Agricultural Research Service (ARS), Parlier, CA (33) and the CCTEA (21), 82 isolates from a collection maintained by ARS in Beltsville, MD (5), and 60 isolates maintained by the BCBR (30). Florida and Beltsville isolates were obtained as desiccated leaf and bark tissue from infected Madam Vinous sweet orange (Citrus sinensis L.). These isolates are listed and described in Table 1 and identified using the following suffixes: CCTEA-, CA-, and P- for California isolates; B- for ARS Beltsville isolates; and FL-, FS-, and T- for Florida isolates.

Selection of strain-specific TaqMan probes. Sequences between P25 (CP) and P27 (CPm) coat protein genes of various isolates were used as previously reported to design a selective $6^{\prime}$ carboxy-fluorescein (6-FAM)-labeled TaqMan probe (CPi-VT3, minor groove binding [MGB]-ACGGKGRTATTRCGC) for the identification of isolates containing VT and T3 genotypes (29). Based on an alignment of multiple $\mathrm{CP}-\mathrm{CPm}$ intergene sequences, two additional strain-specific TaqMan probes, CPi-T36 (MGBACGGTAACATTATACTATCCC) and CPi-T36NS (MGB-ACG GTARTATYATRCCATCCT), were designed at the same nucleotide positions as the CPi-VT3 probe and were used with the primers $\mathrm{P} 27 \mathrm{~F} / \mathrm{P} 27 \mathrm{R}$ (29). The alignment of the multiple sequences and analysis of conserved sequences was carried out using ClustalX (31), followed by the neighbor-joining method in the MegAlign program of DNASTAR (Lasergene, 7.2) to determine phylogenetic relationships.

The CPi-VT3 and CPi-T36 MGB probes were both labeled with 6-FAM and used in Parlier, whereas assays performed in Florida were labeled with NED (Applied Biosystem) and VIC (Applied Biosystem), respectively. The CPi-T36NS probe was labeled with tetrachloro-6-carboxyfluorescein (TET). These strain-specific probes were tested in multiplex reactions with the generic CP25f/r primers and Cyanine 5 (CY5)-labeled TaqMan probe CP-CY5 (28).

Comparison of extraction procedures for qPCR templates. Young leaves, midribs, and bark infected with CTV isolates P29 and P81 were used to evaluate methods for qPCR template preparation. Total nucleic acids (TNA) from $\approx 200 \mathrm{mg}$ of fresh tissue or $50 \mathrm{mg}$ of desiccated tissue were isolated by chromatography on silica particles (28) or by sodium dodecyl sulfate (SDS)-potassium acetate (14) and used directly in one-step realtime RT-PCR assay. Immunocapture with CTV rabbit polyclonal antiserum 1212 (Plant Pathology Department, University of Florida, Gainesville) followed by RT-PCR (12) was tested as an alternative to TNA isolation. In this case, extraction was performed with PBS-PVP-Tween buffer (1:5, wt/vol) and PCR using tubes precoated with the CTV antiserum at a dilution of 1:1,000. Samples processed in Parlier were ground in 2-ml tubes using the Mini-Beadbeater 96 (BioSpec Products, Inc., Bartlesville, OK), whereas in Florida, a Homex 6 with tissue in the manufacturer's plastic bag (Bioreba, Switzerland) was used. Aliquots of each nucleic acid preparation were used to set up qPCR reactions using the CTV generic primer/TaqMan probe set (CP25f/r-CTV-CY5) (28). The resultant cycle threshold $(\mathrm{Ct})$ values were then compared to evaluate the efficacy of each extraction procedure tested.

Single and multiplex real-time RT-PCR assays. One and a half microliters of plant TNA containing $\approx 0.3$ to $0.5 \mu \mathrm{g}$ of total RNA or cDNA prepared as described above was used in a final volume of $12.5 \mu \mathrm{l}$ containing $1 \times$ iScript one-step RT-PCR buffer and iScript reverse transcriptase (for one-step qRT-PCR) (BioRad Laboratories, Hercules, CA) or $1 \times$ iQ Supermix buffer (for qPCR) (Bio-Rad Laboratories). Primer and probe concentrations and cycling conditions for P25F/R-CTV-CY5; P27F/R-CPi-VT3, $\mathrm{CPi}-\mathrm{T} 36$, and $\mathrm{CPi}-\mathrm{T} 36 \mathrm{NS}$ probes were as previously reported for single and multiplex reactions (29). Reactions were performed with the following conditions: $80 \mathrm{nM}$ for CTV-CY5 probe; $160 \mathrm{nM}$ for each CPi probe; $160 \mathrm{nM}$ for P25R; and $320 \mathrm{nM}$ for $\mathrm{P} 25 \mathrm{~F}, \mathrm{P} 27 \mathrm{R}$, and P27F. The amplification profile was one cycle at $55^{\circ} \mathrm{C}$ for $2 \mathrm{~min}$ and then $5 \mathrm{~min}$ at $95^{\circ} \mathrm{C}$ followed by 40 cycles at $95^{\circ} \mathrm{C}$ for $15 \mathrm{~s}$ and $56^{\circ} \mathrm{C}$ for $40 \mathrm{~s}$. The first cycle was skipped when the qPCR followed the cDNA synthesis (two-step qPCR).

Interference of nontarget CTV isolates was tested by spiking a constant quantity of TNA from the P81 isolate (mild, T30 genotype) to each serial dilution of the CPi target isolate. The quantity $(\mu \mathrm{l})$ of P81 added was determined by its Ct value with CTV-CY5 (generic) probe in a way that the first dilution of each target isolate contained an equimolar amount of both isolates (target and nontarget).

The treatments in the multiplex assays were as follows: (A) TNA from P29 (VT genotype) and B3 (T36 genotype) isolates tested with a mixture of CPi-VT3, CPi-T36, and CP-CY5 probes; (B) TNA from B3 and CA-P116 (T36-NS genotype) isolates tested with a mixture of CPi-T36, CPi-T36NS, and CP-CY5 probes; (C) same as treatment A except the TNA from the isolate CA-P116 was added to the mixture along with the CPi-T36NS probe; and (D) all three target strains (P29, B3, and CA-P116) with all $\mathrm{CPi}$ probes without the generic probe.

Treatments A and B were used to test a panel of selected isolates (Table 1); assays were conducted with two replications for each sample, plus a CTV-negative control, a CTV-positive control, and a nontemplate control. Samples from CCTEA and ARS-Beltsville and California field isolates were processed in one-step qRT-PCR, while a two-step qPCR was performed on Florida samples.

\section{RESULTS}

Single and multiplex real-time RT-PCR assays. Excellent CTV detection and strain differentiation were achieved with the probes developed. All nucleic acid preparations tested as templates for qPCR assays were effective. Based on $\mathrm{Ct}$ values, however, the best procedure for nucleic acid extraction was by chromatography on silica particles (range 13.3 to 18.1). The SDSpotassium acetate method resulted in slightly higher $\mathrm{Ct}$ values (range 15.3 to 19.6 ) but was still effective. qPCR using viral cDNA synthesized after immunocapture of virus particles resulted in a higher $\mathrm{Ct}$ value indicating it was less effective than the other two methods.

Standard curves generated in single assays with the CPi-T36 and CPi-T36NS probes had correlation coefficients $\left(R^{2}\right)$ of 0.989 and 0.998 , respectively, indicating high reproducibility and amplification efficiency in the optimal range of 90 to $120 \%$. When these probes were multiplexed with the universal CP-CY5, the $R^{2}$ values of the standard curves generated using the same 10 -fold dilutions ranged from 0.990 for the CP-CY5 to 0.996 for the FAM-labeled CPi-T36 and 0.999 for the TET-labeled CPiT36NS, thus indicating high reproducibility and no reduction in the amplification efficiency. Specifically for the CPi-T36, the efficiency for the single was 91.8 versus 91.7 for the multiplex assays and that for the CPi-T36NS was 95.0 versus 93.2, respectively. The amplification efficiency obtained with the $\mathrm{CP}$ CY5 in these multiplex assays was $120 \%$ versus $111 \%$, respectively.

When the five 10-fold dilutions prepared with the reference isolates B3 and P29 were artificially mixed and used as a template for the multiplex assays, both isolates were clearly detected by the CPi-VT3 and CPi-T36 probes as well as by the generic CP-CY5 probe up to a dilution of $10^{-4}$. No interference was observed when the two probes were labeled with the 6FAM or individually labeled with NED and VIC (data not shown). Similarly, CPi-T36, CPi-T36NS, and CP-CY5 probes simultaneously identified the isolates B3 and CA-P116 in all five mixed 10-fold dilutions. The only exception in performance found was when the CPi-T36NS 
TABLE 1. Isolates used to optimize and validate the multiplex assays ${ }^{\mathrm{a}}$

\begin{tabular}{|c|c|c|c|c|c|c|c|}
\hline \multirow[b]{2}{*}{ Isolate $^{b}$} & \multicolumn{4}{|c|}{$\begin{array}{l}\text { Results of the real-time RT-PCR } \\
\text { (Cycle threshold) }\end{array}$} & \multirow[b]{2}{*}{ Symptom category ${ }^{\mathrm{c}}$} & \multirow{2}{*}{$\begin{array}{l}\text { MCA13 } \\
\text { reactivity }\end{array}$} & \multirow{2}{*}{$\begin{array}{l}\text { Reference for biocharacterization } \\
\text { and MCA13 reaction }\end{array}$} \\
\hline & CP25-CY5 & CPi-VT3 & CPi-T36 & CPi-T36NS & & & \\
\hline & \multicolumn{4}{|c|}{ VT or T3 genotype in single or mixed infection } & & & \\
\hline B23 & 19.00 & 19.04 & 0 & 0 & $\operatorname{GSP}(0.5-3) \&$ & Positive & Hilf et al. (12); Garnsey et al. (5) \\
\hline B31 & 22.09 & 22.81 & 0 & 22.31 & GVIS (1-2.2) & Positive & Hilf et al. (12); Garnsey et al. (5) \\
\hline B46 & 21.95 & 20.38 & 0 & 0 & & Positive & Hilf et al. (12); Garnsey et al. (5) \\
\hline B188 & 20.36 & 24.74 & 0 & 0 & & Positive & Hilf et al. (12); Garnsey et al. (5) \\
\hline B248 & 17.94 & 22.47 & 0 & 0 & & Positive & Hilf et al. (12); Garnsey et al. (5) \\
\hline B343 & 19.86 & 20.86 & 20.33 & 0 & & Positive & Hilf et al. (12); Garnsey et al. (5) \\
\hline B384 & 23.83 & 24.56 & 0 & 0 & & Positive & Hilf et al. (12); Garnsey et al. (5) \\
\hline FL0039 & 22.07 & 20.22 & 23.14 & $\mathrm{nt}^{\mathrm{e}}$ & & Positive & Sieburth et al. (30) \\
\hline FL194 & 22.32 & 19.65 & 22.62 & nt & & Positive & Sieburth et al. (30) \\
\hline FL197 & 23.63 & 20.83 & 25.75 & nt & & Positive & Sieburth et al. (30) \\
\hline FL205 & 29.61 & 27.11 & 0 & 0 & & Positive & Sieburth et al. (30) \\
\hline B8 & 20.51 & 19.87 & 0 & 0 & SY (2-3) & Positive & Hilf et al. (12); Garnsey et al. (5) \\
\hline B119 & 18.97 & 21.97 & 0 & 0 & GSP (1.5-2.5) & Positive & Hilf et al. (12); Garnsey et al. (5) \\
\hline B131 & 18.94 & 24.81 & 0 & 0 & GVIS (2-2.8) & Positive & Hilf et al. (12); Garnsey et al. (5) \\
\hline B136 & 27.97 & 27.17 & 0 & 0 & TBS-WB (1-3) & Positive & Hilf et al. (12); Garnsey et al. (5) \\
\hline B152 & 20.17 & 20.51 & 0 & 0 & & Positive & Hilf et al. (12); Garnsey et al. (5) \\
\hline B211 & 21.16 & 21.78 & 0 & 0 & & Positive & Hilf et al. (12); Garnsey et al. (5) \\
\hline CCTEA106 & 18.95 & 18.45 & 0 & 0 & & Positive & Polek et al. (21) \\
\hline CCTEA107 & 18.65 & 19.56 & 0 & 0 & & Positive & Polek et al. (21) \\
\hline CCTEA99416 & 17.98 & 22.35 & 0 & 20.65 & & Positive & Polek et al. (21) \\
\hline B6 & 18.34 & 18.17 & 0 & 0 & GVIS (1.5-3) & Positive & Hilf et al. (12); Garnsey et al. (5) \\
\hline B28 & 17.88 & 20.18 & 0 & 0 & SY $(0.7-3)$ & Positive & Hilf et al. (12); Garnsey et al. (5) \\
\hline B30 & 20.47 & 20.55 & 0 & 0 & GSP $(0.3-2)$ & Positive & Hilf et al. (12); Garnsey et al. (5) \\
\hline B37 & 18.03 & 19.31 & 0 & 0 & $\operatorname{OSP}(0.7-2.5)$ & Positive & Hilf et al. (12); Garnsey et al. (5) \\
\hline B58 & 21.38 & 22.87 & 0 & 21.03 & & Positive & Hilf et al. (12); Garnsey et al. (5) \\
\hline B65 & 17.63 & 22.31 & 0 & 0 & & Positive & Hilf et al. (12); Garnsey et al. (5) \\
\hline B71 & 17.31 & 17.54 & 0 & 0 & & Positive & Hilf et al. (12); Garnsey et al. (5) \\
\hline B77 & 19.77 & 23.46 & 0 & 24.56 & & Positive & Hilf et al. (12); Garnsey et al. (5) \\
\hline B165 & 20.72 & 20.86 & 0 & 0 & & Positive & Hilf et al. (12); Garnsey et al. (5) \\
\hline B185 & 20.07 & 18.61 & 0 & 24.22 & & Positive & Hilf et al. (12); Garnsey et al. (5) \\
\hline B192AT & 15.04 & 15.27 & 0 & 0 & & Positive & Hilf et al. (12); Garnsey et al. (5) \\
\hline B195 & 18.88 & 18.58 & 0 & 0 & & Positive & Hilf et al. (12); Garnsey et al. (5) \\
\hline B199 & 22.70 & 22.01 & 0 & 0 & & Positive & Hilf et al. (12); Garnsey et al. (5) \\
\hline B219 & 20.37 & 18.31 & 0 & 19.25 & & Positive & Hilf et al. (12); Garnsey et al. (5) \\
\hline B253 & 18.87 & 24.26 & 0 & 0 & & Positive & Hilf et al. (12); Garnsey et al. (5) \\
\hline $\mathrm{B} 255$ & 20.54 & 26.40 & 0 & 24.94 & & Positive & Hilf et al. (12); Garnsey et al. (5) \\
\hline $\mathrm{B} 270$ & 20.13 & 23.28 & 0 & 19.26 & & Positive & Hilf et al. (12); Garnsey et al. (5) \\
\hline B316 & 19.28 & 21.70 & 0 & 25.78 & & Positive & Hilf et al. (12); Garnsey et al. (5) \\
\hline B405 & 17.88 & 20.00 & 0 & 0 & & Positive & Hilf et al. (12); Garnsey et al. (5) \\
\hline B417 & 19.55 & 21.16 & 0 & 18.88 & & Positive & Hilf et al. (12); Garnsey et al. (5) \\
\hline P29 & 15.65 & 17.32 & 0 & 0 & & Positive & R. K. Yokomi, unpublished data \\
\hline P60 & 16.23 & 16.32 & 0 & 0 & & Positive & R. K. Yokomi, unpublished data \\
\hline P108 & 19.36 & 21.32 & 0 & 0 & & Positive & R. K. Yokomi, unpublished data \\
\hline P108-1Bat & 16.32 & 18.56 & 0 & 0 & & Positive & R. K. Yokomi, unpublished data \\
\hline P108-39at & 17.84 & 18.64 & 0 & 0 & & Positive & R. K. Yokomi, unpublished data \\
\hline P109 & 17.18 & 18.39 & 0 & 0 & & Positive & R. K. Yokomi, unpublished data \\
\hline P109-2at & 18.96 & 23.96 & 0 & 0 & & Positive & R. K. Yokomi, unpublished data \\
\hline P109-4at & 19.45 & 15.63 & 0 & 0 & & Positive & R. K. Yokomi, unpublished data \\
\hline FL007 & 22.86 & 20.65 & 0 & nt & SY (0.7-3) & Positive & Sieburth et al. (30) \\
\hline FL208 & 29.73 & 25.79 & 26.49 & nt & GSP $(0.3-2)$ & Positive & Sieburth et al. (30) \\
\hline FS043 & 32.87 & 27.25 & 0 & nt & GVIS (1.5-3) & Positive & Sieburth et al. (30) \\
\hline Т66H548 & 28.35 & 22.37 & 0 & 0 & $\operatorname{OSP}(0.7-2.5)$ & Positive & Sieburth et al. (30) \\
\hline T068 & 26.88 & 24.40 & 0 & 0 & & Positive & Sieburth et al. (30) \\
\hline T068-1 & 25.29 & 27.6 & 0 & 0 & & Positive & Sieburth et al. (30) \\
\hline
\end{tabular}

(Continued on next page)

${ }^{a}$ Isolates in bold were used as reference genotypes to optimize the qRT-PCR protocols and test the strain specificity of the primer/probe sets.

${ }^{\mathrm{b}}$ In each category isolates were listed in the following order: Agricultural Research Service (ARS), Beltsville; Central California Tristeza Eradication Agency (CCTEA) \& ARS, Parlier; and Florida BCBR (Bureau of Citrus Budwood Registration), Florida Department of Agriculture and Consumer Services, Winter Haven, FL.

${ }^{c}$ Biological assays data are from Garsney et al. (5), Polek et al. (21), Sieburth et al. (30), and R. K. Yokomi (unpublished data). Reactions induced in sour orange (SO), Duncan grapefruit (DGF) and Madam Vinous (MV); GSP = stem-pitting on DGF; OSP = stem-pitting on MV; SY = seedling yellows on SO and/or DGF; GVIS= severe stunting on DGF; TBS-WB = thick bark syndrome with wood bristles on DGF $(5,30)$. Numbers in parenthesis indicate the range of the severity scored on a scale of 0 to 3 . For CCTEA isolates, the scored reported have been adjusted from a scale 0 to 15 to a scale of 0 to 3 (21).

${ }^{\mathrm{d}}$ MCA13 reactivity data were from Garsney et al. (5), Hilf et al. (12), Polek et al. (21), Sieburth et al. (30), and R. K. Yokomi (unpublished data).

${ }^{\mathrm{e}} \mathrm{nt}=$ not tested.

${ }^{\mathrm{f}} \mathrm{NA}=$ data not available.

g Infected Madam Vinous plant coinoculated. 
TABLE 1. (Continued from previous page)

\begin{tabular}{|c|c|c|c|c|c|c|c|}
\hline \multirow[b]{2}{*}{ Isolate $^{b}$} & \multicolumn{4}{|c|}{$\begin{array}{l}\text { Results of the real-time RT-PCR } \\
\text { (Cycle threshold) }\end{array}$} & \multirow[b]{2}{*}{ Symptom category $^{c}$} & \multirow{2}{*}{$\begin{array}{l}\text { MCA13 } \\
\text { reactivity }\end{array}$} & \multirow{2}{*}{$\begin{array}{l}\text { Reference for biocharacterization } \\
\text { and MCA13 reaction }\end{array}$} \\
\hline & CP25-CY5 & CPi-VT3 & CPi-T36 & CPi-T36NS & & & \\
\hline B24 & 20.32 & 20.73 & 0 & 0 & SY (1.5) & Positive & Hilf et al. (12); Garnsey et al. (5) \\
\hline B36 & 28.73 & 27.97 & 0 & 0 & $N A^{f}$ & Positive & Hilf et al. (12); Garnsey et al. (5) \\
\hline B148 & 22.17 & 20.99 & 0 & 0 & & Positive & Hilf et al. (12); Garnsey et al. (5) \\
\hline B166 & 21.11 & 19.71 & 0 & 0 & & Positive & Hilf et al. (12); Garnsey et al. (5) \\
\hline B246 & 23.56 & 23.65 & 0 & 20.73 & & Positive & Hilf et al. (12); Garnsey et al. (5) \\
\hline B412 & 20.07 & 23.63 & 0 & 22.65 & & Positive & Hilf et al. (12); Garnsey et al. (5) \\
\hline FL206 & 30.51 & 23.54 & 25.39 & $\mathrm{nt}$ & & Positive & Sieburth et al. (30) \\
\hline FL216 & 28.85 & 27.41 & 0 & 0 & & Positive & Sieburth et al. (30) \\
\hline T003AT10 & 28.44 & 21.45 & 0 & $\mathrm{nt}$ & & Positive & Sieburth et al. (30) \\
\hline B22 & 21.94 & 22.01 & 0 & 0 & Symptomless & Positive & Hilf et al. (12); Garnsey et al. (5) \\
\hline $\mathrm{B} 25$ & 19.67 & 19.76 & 0 & 0 & & Positive & Hilf et al. (12); Garnsey et al. (5) \\
\hline B79 & 22.93 & 23.59 & 0 & 0 & & Positive & Hilf et al. (12); Garnsey et al. (5) \\
\hline FL187 & 26.75 & 21.14 & 21.86 & $\mathrm{nt}$ & & Positive & Sieburth et al. (30) \\
\hline FL192 & 25.75 & 19.62 & 20.91 & nt & & Positive & Sieburth et al. (30) \\
\hline FL207 & 31.39 & 22.47 & 25.26 & nt & & Positive & Sieburth et al. (30) \\
\hline \multirow[t]{2}{*}{ FS595 } & 28.61 & 19.93 & 0 & $\mathrm{nt}$ & & Positive & Sieburth et al. (30) \\
\hline & \multicolumn{4}{|c|}{ Mixture of different genotypes } & & & \\
\hline B192 & 24.78 & 24.21 & 0 & 0 & \multirow[t]{9}{*}{ Symptomless } & Positive & Hilf et al. (12); Garnsey et al. (5) \\
\hline B340 & 21.31 & 20.33 & 0 & 0 & & Positive & Hilf et al. (12); Garnsey et al. (5) \\
\hline FL084 & 20.00 & 0 & 19.67 & $\mathrm{nt}$ & & Positive & Sieburth et al. (30) \\
\hline FL123 & 20.46 & 0 & 20.36 & $\mathrm{nt}$ & & Positive & Sieburth et al. (30) \\
\hline FL129 & 23.32 & 0 & 21.00 & nt & & Positive & Sieburth et al. (30) \\
\hline FS540 & 22.33 & 0 & 21.26 & nt & & Positive & Sieburth et al. (30) \\
\hline FS545 & 21.61 & 0 & 22.74 & nt & & Positive & Sieburth et al. (30) \\
\hline FS625 & 23.39 & 0 & 22.04 & nt & & Positive & Sieburth et al. (30) \\
\hline T067 & 27.52 & 0 & 22.24 & nt & & Positive & Sieburth et al. (30) \\
\hline B271 & 19.13 & 0 & 18.57 & 0 & \multirow[t]{8}{*}{$\operatorname{GSP}(0.3-1.2)$} & Positive & Hilf et al. (12); Garnsey et al. (5) \\
\hline FL079 & 18.54 & 0 & 18.01 & $\mathrm{nt}$ & & Positive & Sieburth et al. (30) \\
\hline FL086 & 17.45 & 0 & 18.43 & nt & & Positive & Sieburth et al. (30) \\
\hline FL149 & 16.81 & 0 & 17.05 & $\mathrm{nt}$ & & Positive & Sieburth et al. (30) \\
\hline FL173 & 23.66 & 0 & 23.06 & $\mathrm{nt}$ & & Positive & Sieburth et al. (30) \\
\hline FL188 & 23.57 & 0 & 22.13 & nt & & Positive & Sieburth et al. (30) \\
\hline FS252 & 27.61 & 0 & 24.67 & $\mathrm{nt}$ & & Positive & Sieburth et al. (30) \\
\hline FS329 & 23.81 & 0 & 22.97 & nt & & Positive & Sieburth et al. (30) \\
\hline FL145 & 18.98 & 0 & 19.19 & $\mathrm{nt}$ & \multirow{2}{*}{$\begin{array}{l}\text { OSP }(0.5) \& \\
\text { GSP (1.3) }\end{array}$} & Positive & Sieburth et al. (30) \\
\hline FL169 & 22.52 & 0 & 21.49 & nt & & Positive & Sieburth et al. (30) \\
\hline FS425 & 27.57 & 0 & 25.19 & $\mathrm{nt}$ & \multirow[t]{6}{*}{ NA } & Positive & Sieburth et al. (30) \\
\hline FS442 & 21.67 & 0 & 21.13 & nt & & Positive & Sieburth et al. (30) \\
\hline FS480 & 19.84 & 0 & 19.84 & nt & & Positive & Sieburth et al. (30) \\
\hline FS642 & 30.30 & 32.68 & 29.73 & 0 & & Positive & Sieburth et al. (30) \\
\hline FS627 & 22.56 & 0 & 21.82 & $\mathrm{nt}$ & & Positive & Sieburth et al. (30) \\
\hline \multirow[t]{2}{*}{ P81+P109g } & 19.11 & 21.89 & 0 & 0 & & Positive & R. K. Yokomi, unpublished data \\
\hline & \multicolumn{4}{|c|}{ Nonstandard genotype } & \multirow{12}{*}{ Symptomless } & & \\
\hline B337 & 28.76 & 0 & 0 & 0 & & Negative & Hilf et al. (12); Garnsey et al. (5) \\
\hline B339 & 24.94 & 0 & 0 & 0 & & Negative & Hilf et al. (12); Garnsey et al. (5) \\
\hline B158 & 26.01 & 0 & 0 & 24.62 & & Positive & Polek et al. (21); R. K. Yokomi, unpublished data \\
\hline CA-P116 & 15.80 & 0 & 0 & 15.65 & & Positive & Polek et al. (21); R. K. Yokomi, unpublished data \\
\hline CCTEA 4 & 21.34 & 0 & 0 & 18.96 & & Positive & Polek et al. (21); R. K. Yokomi, unpublished data \\
\hline CCTEA114 & 23.02 & 0 & 0 & 19.63 & & Positive & Polek et al. (21); R. K. Yokomi, unpublished data \\
\hline CCTEA115 & 19.08 & 0 & 0 & 18.52 & & Positive & Polek et al. (21); R. K. Yokomi, unpublished data \\
\hline CCTEA108 & 23.04 & 0 & 0 & 19.64 & & Positive & Polek et al. (21); R. K. Yokomi, unpublished data \\
\hline CCTEA 89 & 20.36 & 0 & 0 & 17.46 & & Positive & Polek et al. (21); R. K. Yokomi, unpublished data \\
\hline CCTEA 65 & 18.96 & 0 & 0 & 18.96 & & Positive & Polek et al. (21); R. K. Yokomi, unpublished data \\
\hline FL53 & 26.01 & 0 & 0 & 28.16 & & Positive & Sieburth et al. (30) \\
\hline B26 & 26.53 & 0 & 0 & 0 & \multirow[t]{3}{*}{$\operatorname{GSP}(0.2-2.8)$} & Positive & Hilf et al. (12); Garnsey et al. (5) \\
\hline B129 & 24.56 & 0 & 0 & 0 & & Positive & Hilf et al. (12); Garnsey et al. (5) \\
\hline P108-35at & 22.36 & 0 & 0 & 0 & & Positive & R. K. Yokomi, unpublished data \\
\hline B234 & 23.20 & 0 & 0 & 20.49 & NA & NA & Hilf et al. (12); Garnsey et al. (5) \\
\hline B371 & 21.27 & 0 & 0 & 26.52 & & NA & Hilf et al. (12); Garnsey et al. (5) \\
\hline
\end{tabular}

(Continued on next page) 


\begin{tabular}{|c|c|c|c|c|c|c|c|}
\hline \multirow[b]{2}{*}{ Isolate $^{b}$} & \multicolumn{4}{|c|}{$\begin{array}{l}\text { Results of the real-time RT-PCR } \\
\text { (Cycle threshold) }\end{array}$} & \multirow[b]{2}{*}{ Symptom category ${ }^{\mathrm{c}}$} & \multirow{2}{*}{$\begin{array}{l}\text { MCA13 } \\
\text { reactivity }\end{array}$} & \multirow{2}{*}{$\begin{array}{l}\text { Reference for biocharacterization } \\
\text { and MCA13 reaction }\end{array}$} \\
\hline & CP25-CY5 & CPi-VT3 & CPi-T36 & CPi-T36NS & & & \\
\hline & \multicolumn{4}{|c|}{ T30 genotype } & & & \\
\hline B29 & 26.12 & 0 & 0 & 0 & Symptomless & Negative & Hilf et al. (12); Garnsey et al. (5) \\
\hline B32 & 22.41 & 0 & 0 & 0 & & Negative & Hilf et al. (12); Garnsey et al. (5) \\
\hline B252 & 21.81 & 0 & 0 & 0 & & Negative & Hilf et al. (12); Garnsey et al. (5) \\
\hline B277 & 19.33 & 0 & 0 & 0 & & Negative & Hilf et al. (12); Garnsey et al. (5) \\
\hline B305 & 25.60 & 0 & 0 & 0 & & Negative & Hilf et al. (12); Garnsey et al. (5) \\
\hline B334 & 20.24 & 0 & 0 & 0 & & Negative & Hilf et al. (12); Garnsey et al. (5) \\
\hline CCTEA116 & 17.46 & 0 & 0 & 0 & & Negative & Polek et al. (21), R. K. Yokomi, unpublished data \\
\hline CCTEA 165 & 17.87 & 0 & 0 & 0 & & Negative & Polek et al. (21), R. K. Yokomi, unpublished data \\
\hline CCTEA 192 & 19.22 & 0 & 0 & 0 & & Negative & Polek et al. (21), R. K. Yokomi, unpublished data \\
\hline $\mathrm{P} 1$ & 15.36 & 0 & 0 & 0 & & Negative & Polek et al. (21), R. K. Yokomi, unpublished data \\
\hline P100 & 21.33 & 0 & 0 & 0 & & Negative & Polek et al. (21), R. K. Yokomi, unpublished data \\
\hline FL186 & 23.59 & 0 & 0 & nt & & Negative & Sieburth et al. (30) \\
\hline FL193 & 26.13 & 0 & 0 & 0 & & Negative & Sieburth et al. (30) \\
\hline FL230 & 25.68 & 0 & 0 & nt & & Negative & Sieburth et al. (30) \\
\hline FL248 & 25.73 & 0 & 0 & nt & & Negative & Sieburth et al. (30) \\
\hline FL277 & 24.57 & 0 & 0 & nt & & Negative & Sieburth et al. (30) \\
\hline FL311 & 21.32 & 0 & 0 & nt & & Negative & Sieburth et al. (30) \\
\hline FL328 & 23.52 & 0 & 0 & $\mathrm{nt}$ & & Negative & Sieburth et al. (30) \\
\hline FS253 & 26.48 & 0 & 0 & 0 & & Negative & Sieburth et al. (30) \\
\hline T026 & 21.93 & 0 & 0 & nt & & Negative & Sieburth et al. (30) \\
\hline T030 & 26.52 & 0 & 0 & 0 & & Negative & Sieburth et al. (30) \\
\hline T55-1 & 25.56 & 0 & 0 & nt & & Negative & Sieburth et al. (30) \\
\hline T069 & 32.38 & 0 & 0 & nt & & Negative & Sieburth et al. (30) \\
\hline T069-1 & 29.97 & 0 & 0 & nt & & Negative & Sieburth et al. (30) \\
\hline B314 & 20.13 & 0 & 0 & 0 & & Negative & Hilf et al. (12); Garnsey et al. (5) \\
\hline B391 & 21.37 & 0 & 0 & 0 & $\begin{array}{l}\text { Mild DGF reactions } \\
\text { (GSP } 0.1-0.8) ; \text { OSP } \\
\quad(0.3-0.5)\end{array}$ & Negative & Hilf et al. (12); Garnsey et al. (5) \\
\hline B397 & 20.83 & 0 & 0 & 0 & & Negative & Hilf et al. (12); Garnsey et al. (5) \\
\hline CCTEA 117 & 15.32 & 0 & 0 & 0 & & Negative & Polek et al. (21), R. K. Yokomi, unpublished data \\
\hline CCTEA 118 & 21.39 & 0 & 0 & 0 & & Negative & Polek et al. (21), R. K. Yokomi, unpublished data \\
\hline CCTEA 120 & 19.87 & 0 & 0 & 0 & & Negative & Polek et al. (21), R. K. Yokomi, unpublished data \\
\hline CCTEA 127 & 18.75 & 0 & 0 & 0 & & Negative & Polek et al. (21), R. K. Yokomi, unpublished data \\
\hline CCTEA 128 & 23.56 & 0 & 0 & 0 & & Negative & Polek et al. (21), R. K. Yokomi, unpublished data \\
\hline CCTEA 137 & 16.49 & 0 & 0 & 0 & & Negative & Polek et al. (21), R. K. Yokomi, unpublished data \\
\hline CCTEA 139 & 16.89 & 0 & 0 & 0 & & Negative & Polek et al. (21), R. K. Yokomi, unpublished data \\
\hline CCTEA 141 & 18.19 & 0 & 0 & 0 & & Negative & Polek et al. (21), R. K. Yokomi, unpublished data \\
\hline CCTEA 146 & 19.23 & 0 & 0 & 0 & & Negative & Polek et al. (21), R. K. Yokomi, unpublished data \\
\hline CCTEA 150 & 18.53 & 0 & 0 & 0 & & Negative & Polek et al. (21), R. K. Yokomi, unpublished data \\
\hline CCTEA 154 & 17.69 & 0 & 0 & 0 & & Negative & Polek et al. (21), R. K. Yokomi, unpublished data \\
\hline CCTEA 155 & 18.33 & 0 & 0 & 0 & & Negative & Polek et al. (21), R. K. Yokomi, unpublished data \\
\hline CCTEA 160 & 20.06 & 0 & 0 & 0 & & Negative & Polek et al. (21), R. K. Yokomi, unpublished data \\
\hline CCTEA 161 & 17.58 & 0 & 0 & 0 & & Negative & Polek et al. (21), R. K. Yokomi, unpublished data \\
\hline CCTEA 162 & 20.01 & 0 & 0 & 0 & & Negative & Polek et al. (21), R. K. Yokomi, unpublished data \\
\hline CCTEA 163 & 22.31 & 0 & 0 & 0 & & Negative & Polek et al. (21), R. K. Yokomi, unpublished data \\
\hline CCTEA 170 & 19.64 & 0 & 0 & 0 & & Negative & Polek et al. (21), R. K. Yokomi, unpublished data \\
\hline CCTEA 172 & 18.46 & 0 & 0 & 0 & & Negative & Polek et al. (21), R. K. Yokomi, unpublished data \\
\hline CCTEA 175 & 21.84 & 0 & 0 & 0 & & Negative & Polek et al. (21), R. K. Yokomi, unpublished data \\
\hline CCTEA 180 & 18.32 & 0 & 0 & 0 & & Negative & Polek et al. (21), R. K. Yokomi, unpublished data \\
\hline CCTEA 183 & 15.26 & 0 & 0 & 0 & & Negative & Polek et al. (21), R. K. Yokomi, unpublished data \\
\hline CCTEA 197 & 18.52 & 0 & 0 & 0 & & Negative & Polek et al. (21), R. K. Yokomi, unpublished data \\
\hline CCTEA 193 & 16.00 & 0 & 0 & 0 & & Negative & Polek et al. (21), R. K. Yokomi, unpublished data \\
\hline CCTEA 199 & 17.03 & 0 & 0 & 0 & & Negative & Polek et al. (21), R. K. Yokomi, unpublished data \\
\hline CCTEA 96235 & 18.65 & 0 & 0 & 0 & & Negative & Polek et al. (21), R. K. Yokomi, unpublished data \\
\hline CCTEA 96112 & 18.91 & 0 & 0 & 0 & & Negative & Polek et al. (21), R. K. Yokomi, unpublished data \\
\hline P81 & 18.90 & 0 & 0 & 0 & & Negative & Polek et al. (21), R. K. Yokomi, unpublished data \\
\hline FL204 & 25.02 & 0 & 0 & nt & & Negative & Sieburth et al. (30) \\
\hline FL209 & 27.23 & 0 & 0 & 0 & & Negative & Sieburth et al. (30) \\
\hline FL217 & 25.67 & 0 & 0 & nt & & Negative & Sieburth et al. (30) \\
\hline FL223 & 22.82 & 0 & 0 & $\mathrm{nt}$ & & Negative & Sieburth et al. (30) \\
\hline FL224 & 25.44 & 0 & 0 & $\mathrm{nt}$ & & Negative & Sieburth et al. (30) \\
\hline FL227 & 28.01 & 0 & 0 & nt & & Negative & Sieburth et al. (30) \\
\hline FL237 & 22.48 & 0 & 0 & nt & & Negative & Sieburth et al. (30) \\
\hline FL245 & 22.92 & 0 & 0 & nt & & Negative & Sieburth et al. (30) \\
\hline FL251 & 23.54 & 0 & 0 & $\mathrm{nt}$ & & Negative & Sieburth et al. (30) \\
\hline FL267 & 27.14 & 0 & 0 & nt & & Negative & Sieburth et al. (30) \\
\hline FL272 & 23.92 & 0 & 0 & nt & & Negative & Sieburth et al. (30) \\
\hline FL298 & 27.50 & 0 & 0 & nt & & Negative & Sieburth et al. (30) \\
\hline
\end{tabular}


and CPi-VT3 probes were combined (treatments $\mathrm{C}$ and D), creating an interference which led to the inability to detect isolate CA-P116 (data not shown). This, however, was not the case for the other target isolates. The sensitivity of the multiplex assays (treatments A and B) was as good as that obtained in single reactions. The limit of detection of the multiplex assays were comparable to that of the single (up to $10^{-4}$ ), and the overall $\mathrm{Ct}$ value difference of the multiplex versus the single ranged between $+0.8 /+1.6 \mathrm{Ct}$. In addition, the three CPi probes clearly identified the target sequences up to a ratio of 1:1,000 in the presence of the nontarget P81 mild isolate (Fig. 1).

The three CPi probes effectively detected VT, T3, T36, and T36NS genotypes in single and natural mixed infections from reference collections (Table 1). Panel tests using various CPi probes were in good agreement with the previous work describing the biocharacterization and sequence grouping using MMM markers of each isolate and genotype of this panel (12). All isolates that reacted with the $\mathrm{CPi}$ probes were also found to be positive with the monoclonal antibody MCA13 $(12,20)$ in doubleantibody sandwich indirect (DASI)-ELISA.

Five budwood source trees from the Florida BCBR tested positive with the CPi-T36 probe but failed to react with MCA13 in DASI-ELISA (Table 2) (P. J. Sieburth, unpublished data) and suggests a higher sensitivity of the qPCR assays compared with ELISA.

CPi-T36NS probe. CPi-T36NS probe detected isolates which did not react with any of the MMM markers; however, for a few isolates faint amplicons and inconsistent results were obtained with T30POL primers. These isolates were also associated with lower MCA13 reactivity $\left(\mathrm{OD}_{405}\right)$ than T36 or VT isolates in DASI-ELISA (R. K. Yokomi, unpublished data). The low MCA13 reactivity of these isolates resulted in occasional false negatives in detecting field infections by serology compared with their reactivity with the CPi-T36NS probe (Table 2). For some of these isolates the presence of the MCA13 epitope was confirmed by sequence analysis of the CP gene (Genbank accession nos. EU878380, EU325938, EU325936, EU325930, and GQ131683). This group included five isolates in the CCTEA collection (CCTEA65, 89, 108, 114, and 115) and some more widely distributed isolates from southern California (Table 2). CCTEA biological indexing of representative isolates indicated that these isolates were mild in all indicator plants (21). A few of these isolates were detected in the Beltsville collection where they were primarily in mixed infections with severe VT or T3 strains. This isolate type was also found among the Florida isolates (FL053).

The CPi probes did not react with any T30 genotype isolates of a panel in Table 1 (12) or with any from an outgroup of isolates including but not limited to P108-35at, B26, B129, and 99416-9 (Fig. 1).

\section{DISCUSSION}

Although there is great genetic diversity and variability between and among various CTV isolates $(1,16,32)$, no clear association of specific CTV-induced symptoms exits with specific genotypes. Variable pathogenicity occurs among isolates sharing the same genotype $(11,12)$. However, mild to moderate symptoms are typically associated with T30 genotypes while T3, VT, and T36 genotypes are associated with more severe symptoms $(1,12)$. Our objective was to develop a reliable and sensitive assay to identify isolates that have potential economic impact in commercial citrus groves. Three qRT-PCR TaqMan probes called CPi-T36, CPiT36NS, and CPi-VT3 were designed to target isolates in three distinct phylogenetic groups (Fig. 1). Although qPCR reactivity was based on short sequences of the MGB-TaqMan probe (15 to $21 \mathrm{nt}$ ), differentiation of strains of a large panel of genetic diverse isolates was confirmed.

TABLE 1. (Continued from previous page)

\begin{tabular}{|c|c|c|c|c|c|c|c|}
\hline \multirow[b]{2}{*}{ Isolate $^{\mathrm{b}}$} & \multicolumn{4}{|c|}{$\begin{array}{l}\text { Results of the real-time RT-PCR } \\
\text { (Cycle threshold) }\end{array}$} & \multirow[b]{2}{*}{ Symptom category $^{c}$} & \multirow{2}{*}{$\begin{array}{l}\text { MCA13 } \\
\text { reactivity }\end{array}$} & \multirow{2}{*}{$\begin{array}{c}\text { Reference for biocharacterization } \\
\text { and MCA13 reaction }\end{array}$} \\
\hline & CP25-CY5 & CPi-VT3 & CPi-T36 & CPi-T36NS & & & \\
\hline FL302 & 17.40 & 0 & 0 & nt & & Negative & Sieburth et al. (30) \\
\hline $\mathrm{T} 4$ & 17.13 & 0 & 0 & 0 & & Negative & Sieburth et al. (30) \\
\hline \multirow[t]{2}{*}{ FL278 } & 27.38 & 0 & 0 & nt & OSP (1.5) GSP (3) & Negative & Sieburth et al. (30) \\
\hline & \multicolumn{4}{|c|}{ T36 genotype } & & & \\
\hline B3 & 15.32 & 0 & 14.58 & 0 & SY (1.5-2) & Negative & Hilf et al. (12); Garnsey et al. (5) \\
\hline CCTEA96337 & 18.36 & 0 & 16.19 & 0 & & Negative & Polek et al. (21) \\
\hline CCTEA96339 & 20.50 & 0 & 18.89 & 0 & & Negative & Polek et al. (21) \\
\hline FL092 & 19.39 & 0 & 19.16 & nt & & Negative & Sieburth et al. (30) \\
\hline FL139 & 22.00 & 0 & 21.02 & nt & & Negative & Sieburth et al. (30) \\
\hline T036 & 24.79 & 0 & 20.17 & 0 & & Negative & Sieburth et al. (30) \\
\hline T066 & 29.62 & 0 & 24.65 & 0 & & Negative & Sieburth et al. (30) \\
\hline T066-1 & 23.09 & 0 & 20.10 & 0 & & Negative & Sieburth et al. (30) \\
\hline T067-1 & 30.08 & 0 & 23.76 & nt & GSP (0.3-1) & Negative & Sieburth et al. (30) \\
\hline FL154 & 20.13 & 0 & 19.99 & nt & & Negative & Sieburth et al. (30) \\
\hline FL165 & 20.33 & 0 & 18.17 & $\mathrm{nt}$ & & Negative & Sieburth et al. (30) \\
\hline FL191 & 22.18 & 0 & 20.83 & $\mathrm{nt}$ & & Negative & Sieburth et al. (30) \\
\hline FS577-1 & 24.38 & 0 & 21.89 & $\mathrm{nt}$ & & Negative & Sieburth et al. (30) \\
\hline T6671P1A & 23.47 & 0 & 21.76 & $\mathrm{nt}$ & & Negative & Sieburth et al. (30) \\
\hline B83 & 24.32 & 0 & 0 & 0 & & Negative & Hilf et al. (12); Garnsey et al. (5) \\
\hline FL104 & 20.87 & 0 & 20.64 & nt & OSP $(0.5-1.5) \&$ & Negative & Sieburth et al. (30) \\
\hline FL141 & 17.41 & 0 & 18.67 & nt & GSP $(0.5)$ & Negative & Sieburth et al. (30) \\
\hline FL090 & 19.58 & 0 & 25.48 & $\mathrm{nt}$ & NA & Negative & Sieburth et al. (30) \\
\hline FL252 & 18.56 & 0 & 19.65 & $\mathrm{nt}$ & & Negative & Sieburth et al. (30) \\
\hline FS377 & 22.70 & 0 & 19.36 & nt & & Negative & Sieburth et al. (30) \\
\hline FS393 & 22.62 & 0 & 21.24 & nt & & Negative & Sieburth et al. (30) \\
\hline FS420 & 23.68 & 0 & 20.10 & $\mathrm{nt}$ & & Negative & Sieburth et al. (30) \\
\hline FS577 & 24.91 & 0 & 22.08 & $\mathrm{nt}$ & & Negative & Sieburth et al. (30) \\
\hline
\end{tabular}


Real-time PCR is a powerful tool for rapid and sensitive detection of plant pathogens and its use for CTV detection and genotype differentiation has been recently reported $(2,22,26-28)$. Ruiz-Ruiz et al. (27) used real-time RT-PCR to differentiate between mild and severe CTV strains. Their method allowed the detection and the identification of the CTV strains by performing four independent reactions. In contrast, the method described herein is based on a multiplex assay that using different primers/ probes with amplification protocols that allowed single tube, simultaneous differentiation of CTV isolates containing VT, T3, or T36 genotypes. Strain differentiation was also extended within the T36 clade to separate a subgroup of MCA13 reactive isolates which are biologically mild and not previously recognized.

The assays herein described were effective in identifying target isolates in a mixture with nontarget isolates. Ruiz-Ruiz et al. (27) were able to detect the severe target genotypes (VT, T3, and T36) in mixtures containing $2.5 \%$ of the homologous and $97.5 \%$ of the heterologous transcripts. Our CPi probes were able to detect the homologous viral RNA up to a ratio of 1:1,000 when mixed with the mild P81 isolate. An example of this sensitivity is the reaction
A
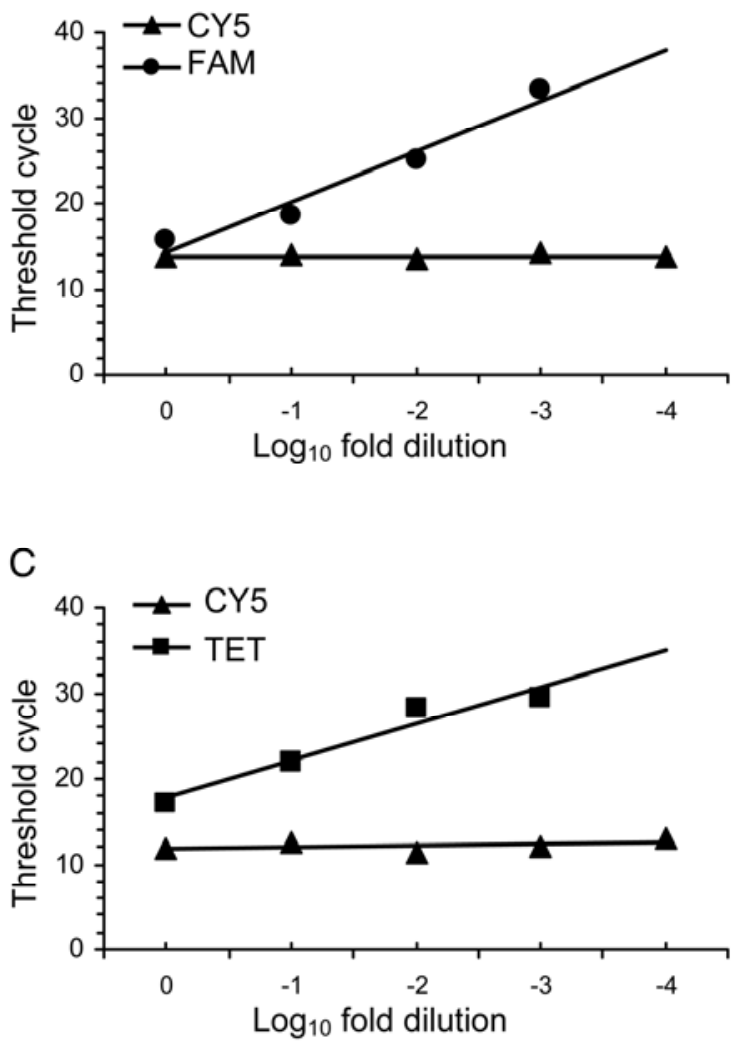

B

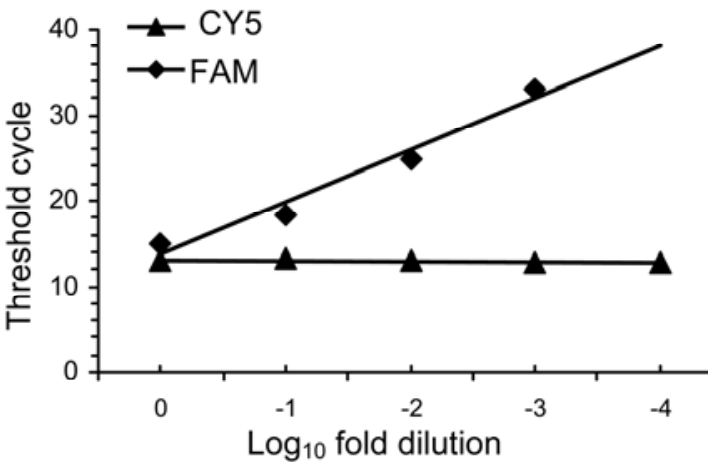

Fig. 1. Standard curves obtained in multiplex real-time reverse transcription-polymerase chain reaction assays with five 10 -fold dilutions (from 1 to $10^{-4}$ ) of total nucleic acids (TNA) from Citrus tristeza virus infected plant P29, CA-P116, and B3, each dilution included a standard amount of TNA recovered from a P81infected plant. A, Standard curve generated with the 10-fold dilutions of P29 TNA using the FAM-labeled CPi-VT3 and the CY5-labeled CP-CY5 probes. B, Standard curve generated with the 10-fold dilutions of B3 TNA using the FAM-labeled Ci-T36 and the CY5-labeled CP-CY5 probes. C, Standard curves generated with the 10-fold dilution of CA-P116 TNA using the FAM-labeled CPi-T36NS and the CY5-labeled CP-CY5 probes. The standard curves are indicated in the graphs by the fluorophore labeling the respective probe. As shown, all three CPi probes detected the target strains up to a dilution of 1.00E-03. Since all dilutions contained an equal amount of the TNA of P81 isolate, constant $\mathrm{Ct}$ values were obtained for all dilutions with the generic CP-CY5 probe.

TABLE 2. Field samples collected in different California plots and from the budwood source trees in Florida during 2008 to $2009^{\mathrm{a}}$

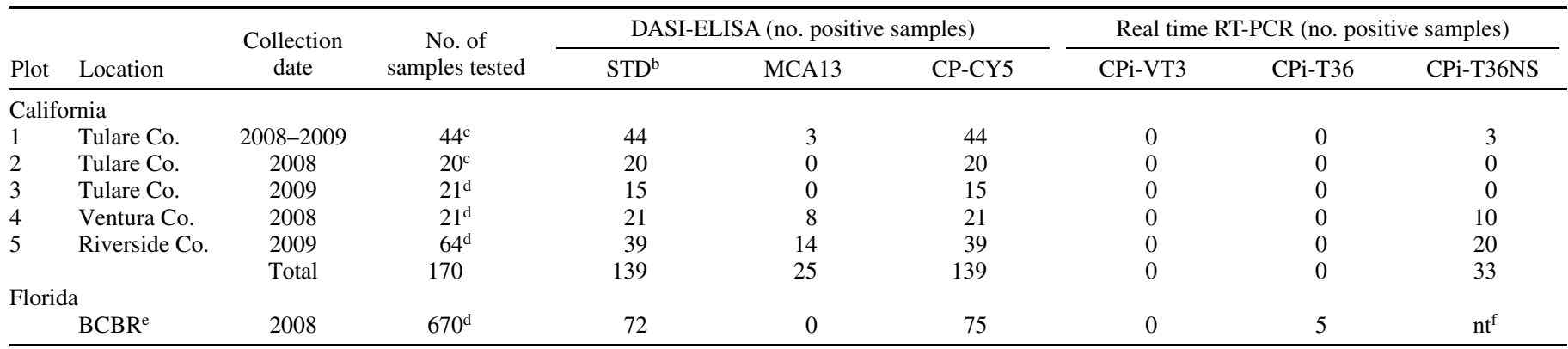

a All samples were tested both in double-antibody sandwich indirect enzyme-linked immunosorbent assay (DASI-ELISA) and real-time reverse transcriptionpolymerase chain reaction (RT-PCR).

${ }^{\mathrm{b}}$ DASI-ELISA performed using a broad-spectrum polyclonal antiserum.

${ }^{c}$ Samples collected from only known Citrus tristeza virus (CTV)-infected trees.

${ }^{\mathrm{d}}$ Samples collected from blocks of trees of unknown CTV status.

${ }^{e}$ BCBR: Bureau of Citrus Budwood Registration, Florida Department of Agriculture and Consumer Services, Winter Haven, FL.

${ }^{\mathrm{f}}$ Not tested. 
of CPi-VT3 probe with the isolate B192, a mixture of mild and VT strains (3). This VT subisolate was not detectable using the SP LNA TaqMan probe (27).

$\mathrm{Ct}$ values based on serial dilutions of TNA from T36, T36NS, and VT genotype isolates had comparable sensitivity by multiplex and single assays. This indicates the assays were optimized and that only minimal interference and/or competition occurred between primer/probe sets.

CPi-T36NS probe successfully detected MCA13-reactive isolates in the collections (CCTEA89, CCTEA65, CCTEA4, CCTEA115, CCTEA114; CCTEA108; B158; FL053) which induced mild symptoms in the indicator plants $(5,21,30)$ and those in field samples which remained undetectable using the MCA13. Nucleotide sequence homology was found among this group of isolates and New Zealand CTV strains recently characterized as Poncirus trifoliata-resistance breaking (RB) isolates (10; S. J. Harper, personal communication) (i.e., NZRB-TH30; Fig. 1). Based on these similarities, RB isolates could be detected by the CPi-T36NS probe. Because the homology of the putative California and New Zealand RB isolates is based on a very small genome region, these California isolates need to be tested for the ability to replicate in $P$. trifoliata.

In conclusion, the TaqMan RT-PCR assays described herein allow detection of CTV in sample extracts from citrus trees. Furthermore, a positive reaction with one or more of the CPi probes identifies the presence of potential virulent virus strains. If a multiplex qRT-PCR test is used for CTV strain differentiation, the CTV-CY5 probe can serve as a CTV-positive internal control to insure that template preparation and qPCR conditions were correct. Finally, the CPi probes differentiated MCA13-reactive isolates into at least three distinct groups which will be useful to separate MCA13-reactive isolates into potentially mild versus potentially more severe isolates.

\section{ACKNOWLEDGMENTS}

M. Saponari was a visiting scientist with the USDA, ARS, PWA, Crop Diseases, Pest and Genetics Research (CDPG), Parlier, CA. This work was funded in part through the Short Term Mobility grant in 2006 and 2008 from the Consiglio Nazionale per le Ricerche (CNR)-Italy. We acknowledge the field and technical assistance of R. DeBorde (USDA, ARS, PWA, CDPG, Parlier, CA). We thank M. Polek (CCTEA, Tulare, CA), J. Hartung, and C. Paul (USDA, ARS, Beltsville, MD) for providing access to CTV collections and S. Garnsey (USDA, ARS, retired) for descriptions of Beltsville and Florida isolates.

\section{LITERATURE CITED}

1. Ayllón, M. A., López, C., Navas-Castillo, J., Garnsey, S. M., Guerri, J., Flores, R., and Moreno P. 2001. Polymorphism of the $5^{\prime}$ terminal region of Citrus tristeza virus (CTV) RNA: Incidence of three sequence types in isolates of different origin and pathogenicity. Arch. Virol. 146:27-40.

2. Bertolini, E., Moreno, A., Capote, N., Olmos, A., De Luis, A., Vidal, E., Pérez-Panadés, J., and Cambra, M. 2007. Quantitative detection of Citrus tristeza virus in plant tissues and single aphids by real-time RT-PCR. Eur. J. Plant Pathol. 120(2):177-188.

3. Brlansky, R. H., Damsteegt, V. D., Howd, D. S., and Roy, A. 2003. Molecular analyses of Citrus tristeza virus subisolates separated by aphid transmission. Plant Dis. 87:397-401.

4. Cevik, B., Pappu, S. S., Pappu, H. R. Benscher, D., Irey, M., Lee, R. F., and Niblett, C. L. 1996. Application of bi-directional PCR to Citrus tristeza virus: Detection and strain differentiation. Pages 17-24 in: Proc. 13th Conf. IOCV. IOCV, Riverside, CA.

5. Garnsey, S. M., Civerolo, E. L., Gumpf, D. J., Paul, C., Hilf, M., Lee, R. F., Brlansky, R. H., Yokomi, R. K., and Hartung, J. S. 2005. Biocharacterization of an international collection of Citrus tristeza virus (CTV) isolates. Pages 75-93 in: Proc. 16th Conf. IOCV. IOCV, Riverside, CA.

6. Genç, H. 2005. A new method for the detection of minor populations of Citrus tristeza virus strains infecting single citrus trees. Turk. J. Agric. For. 29:449-459.

7. Gillings, M., Broadbent, P., Indsto, J., and Lee, R. F. 1993. Characterization of isolates and strains of citrus tristeza closterovirus using restric- tion analysis of the coat protein gene amplified by the polymerase chain reaction. J. Virol. Methods 44:305-317.

8. Gowda, S., Ayllón, M. A., Satyanarayana, T., Bar-Joseph, M., and Dawson, W. O. 2003. Transcription strategy in a Closterovirus: A novel 5'-proximal controller element of Citrus tristeza virus produces 5' - and 3'terminal subgenomic RNAs and differs from 3' open reading frame controller elements. J. Virol. 77:340-352.

9. Halbert, S. E., Genç, H., Cevik, B., Brown, L. G., Rosales, I. M., Manjunath, K. L., Pomerinke, M., Davison, D. A., and Lee, R. F. 2004. Distribution and characterization of Citrus tristeza virus in South Florida following the establishment of Toxoptera citricida. Plant Dis. 88:935-941.

10. Hilf, M. E. 2005. Partial sequence characterization of Citrus tristeza virus associated with breaking of the general resistance to CTV expressed in Poncirus trifoliata. Pages 52-60 in: Proc. 16th Conf. IOCV. IOCV, Riverside, CA.

11. Hilf, M. E., and Garnsey, S. M. 2002. Citrus tristeza virus in Florida: A synthesis of historical and contemporary biological, serological and genetic data. Pages 13-19 in: Proc. 15th Conf. IOCV. IOCV, Riverside, CA.

12. Hilf, M. E., Mavrodieva, V. A., and Garnsey, S. M. 2005. Genetic marker analysis of a global collection of isolates of Citrus tristeza virus: Characterization and distribution of CTV genotypes and association with symptoms. Phytopathology 95:909-917.

13. Huang, Z., Rundell, A. P.,Guan, X., and Powell, C. A. 2004. Detection and isolate differentiation of Citrus tristeza virus in infected field trees based on reverse transcription-polymerase chain reaction. Plant Dis. 88:625-629.

14. Irey, M. S., Gast, T., and Gottwald, T. 2006. Comparison of visual assessment and polymerase chain reaction assay testing to estimate the incidence of the Huanglongbing pathogen in commercial Florida citrus. Proc. Fla. State Hortic. Soc. 119:89-93.

15. Kong, P., Rubio, L., Polek, M., and Falk, B.W. 2000. Population structure and genetic diversity within California Citrus tristeza virus (CTV) isolates. Virus Genes 21:139-145.

16. López, C., Ayllón, M. A., Navas-Castillo, J., Guerri, J., Moreno, P., and Flores, R. 1998. Sequence polymorphism in the $5^{\prime}$ and $3^{\prime}$ terminal regions of Citrus tristeza virus RNA. Phytopathology 88:685-691.

17. Mawassi, M., Karasev, A. V., Mietkiewska, E., Gafny, R., Lee, R. F., Dawson, W. O., and Bar-Joseph, M. 1995. Defective RNA molecules associated with Citrus tristeza virus. Virology 208:383-387.

18. McClean, A. D. P. 1963. Seedling yellows in South African citrus trees. S. Afr. Agric. Sci. 3:259-279.

19. Nikolaeva, O. V., Karasev, A. V., Garnsey, S. M., and Lee, R. F. 1998. Serological differentiation of the Citrus tristeza virus isolates causing stem pitting in sweet orange. Plant Dis. 82:1276-1280.

20. Permar, T. A., Garnsey, S. M., Gumpf, D. J., and Lee, R. F. 1990. A monoclonal antibody that discriminates strains of Citrus tristeza virus. Phytopathology 80:224-228.

21. Polek, M., Gumpf, D. J., Wallen, C. M., and Riley, K. M. 2005. Biological characterization of naturally occurring Citrus tristeza virus strain in California citrus. Pages 68-74 in: Proc. 16th Conf. IOCV. IOCV, Riverside, CA.

22. Rosa, C., Polek, M., Falk, B. W., and Rowhani, A. 2007. Improved efficiency for quantitative and qualitative indexing for Citrus tristeza virus and Citrus psorosis virus. Plant Dis. 91:1089-1095.

23. Rubio, L., Ayllon, M. A., Guerri, J., Pappu, H. R., Niblett, C. L., and Moreno, P. 1996. Differentiation of Citrus tristeza virus (CTV) isolates by single-strand conformation polymorphism analysis of the coat protein gene. Ann. App. Biol. 129:479-489.

24. Rubio, L., Ayllón, M. A., Kong, P., Fernández, A., Polek, M., Guerri, J., Moreno, P., and Falk, B. W. 2001. Genetic variation of Citrus tristeza virus isolates from California and Spain: Evidence for mixed infections and recombination. J. Virol. 75:8054-8062.

25. Rubio, L., Guerri, J., and Moreno, P. 2000. Characterization of Citrus tristeza virus isolates by single strand conformation polymorphism analysis of DNA complementary to their RNA population. Pages 12-17 in: Proc. 16th Conf. IOCV. IOCV, Riverside, CA.

26. Ruiz-Ruiz, S., Moreno, P., Guerri, J., and Ambrós, S. 2007. A real-time RT-PCR assay for detection and absolute quantitation of Citrus tristeza virus in different plant tissues. J. Virol. Methods 145:96-105.

27. Ruiz-Ruiz, S., Moreno, P., Guerri, J., and Ambrós, S. 2009. Discrimination between mild and severe Citrus tristeza virus isolates with a rapid and highly specific real-time reverse transcription-polymerase chain reaction method using TaqMan LNA probes. Phytopathology 99:307-315.

28. Saponari, M., Keremane, M. L., and Yokomi, R. K. 2008. Quantitative detection of Citrus tristeza virus (CTV) in citrus and aphids by real-time reverse transcription-PCR (TaqMan). J. Virol. Methods 147:43-53.

29. Saponari, M., and Yokomi, R. K. Use of the coat protein (CP) and minor $\mathrm{CP}$ intergene sequence to discriminate severe strains of Citrus tristeza virus (CTV) in three U.S. CTV isolate collections. Proc. 17th Conf. 
IOCV. IOCV, Riverside, CA. (In press.)

30. Sieburth, P. J., Nolan, K. F., Hilf, M. E., Lee, R. F., Moreno, P., and Garnsey, S. M. 2005. Discrimination of stem-pitting from other isolates of Citrus tristeza virus. Pages 1-12 in: Proc. 16th Conf. IOCV. IOCV, Riverside, CA.

31. Thompson, J. D., Gibson, T. J., Plewniak, F., Jeanmougin, F., Higgins, D. G. 1997. The ClustalX windows interface: Flexible strategies for multiple sequence alignment aided by quality analysis tools. Nucleic Acids Res. 24:4876-4882.

32. Vives, M. C., Rubio, L., Sambade, A., Mirkov, T. E., Moreno, P., and Guerri, J. 2005. Evidence of multiple recombination events between two
RNA sequence variants within a Citrus tristeza virus isolate. Virology 331:232-237.

33. Yokomi, R. K., and Deborde, R. L. 2005. Incidence, transmissibility, and genotype analysis of Citrus tristeza virus (CTV) isolates from CTV eradicative and noneradicative districts in Central California. Plant Dis. 89:859-866.

34. Yokomi, R. K., Polek, M., Grafton-Cardwell, E. E., Vidalakis, G., O'Connell, N., and Saponari, M. Assessment of the Citrus tristeza virus isolates detected in spring 2007 at the Lindcove Research and Extension Center, Exeter, California. Proc. 17th Conf. IOCV. IOCV, Riverside, CA. (In press.) 